\title{
Estimating the Shapes of Gravity Sources through Optimized Support Vector Classifier (SVC)
}

\author{
Mohammad E. HEKMATIAN ${ }^{1,2}$, Vahid E. ARDESTANI ${ }^{3}$, \\ Mohammad A. RIAHI ${ }^{3}$, Ayyub M.K. BAGH ${ }^{2,4}$, and Jalal AMINI ${ }^{5}$ \\ ${ }^{1}$ Faculty of Basic Sciences of Science and Research Branch, Islamic Azad \\ University, Tehran, Iran; e-mail: mhekmatian@aeoi.org.ir (corresponding author) \\ ${ }^{2}$ Nuclear Fuel Cycle Research School of Nuclear Science \\ and Technology Research Institute (NSTRI), Tehran, Iran \\ ${ }^{3}$ Institute of Geophysics, University of Tehran, Tehran, Iran \\ e-mails: ebrahimz@ut.ac.ir,mariahi@ut.ac.ir \\ ${ }^{4}$ Faculty of Engineering of South Tehran Branch, Islamic Azad University, \\ Tehran, Iran; e-mail: amemar@aeoi.org.ir \\ ${ }^{5}$ Faculty of Engineering, University of Tehran, Tehran, Iran; e-mail: jamini@ut.ac.ir
}

\section{Abstract}

In gravity interpretation methods, an initial guess for the approximate shape of the gravity source is necessary. In this paper, the support vector classifier (SVC) is applied for this duty by using gravity data. It is shown that using SVC leads us to estimate the approximate shapes of gravity sources more objectively. The procedure of selecting correct features is called feature selection (FS).

In this research, the proper features are selected using inter/intra class distance algorithm and also FS is optimized by increasing and decreasing the number of dimensions of features space. Then, by using the proper features, SVC is used to estimate approximate shapes of sources from the six possible shapes, including: sphere, horizontal cylinder, vertical cylinder, rectangular prism, syncline, and anticline. SVC is trained using 300 synthetic gravity profiles and tested by 60 other synthetic and some real gravity profiles (related to a well and two ore bodies), and shapes of their sources estimated properly.

Key words: gravity sources shapes, SVC, feature, gravity profile, FS. 


\section{INTRODUCTION}

Usual methods for estimation of gravity source shape are the forward method and the non linear inversion method. However, for making the shape estimation of gravity source, both of them need to have an initial assumption of the shape of the gravity source. There is no usual method for making the initial assumption of shape of gravity source and practically this assumption is prepared by non-gravity (for example geological) data. An attempt to estimate an approximate shape of gravity sources (as the initial assumption) using artificial neural network was done by Gret and Klingele (1998). Support vector classifier (SVC) algorithm could be used to estimate approximate shapes of gravity sources more objectively. SVC is one of the pattern recognition (PR) algorithms. When the input values (or objects) are different and these values have different labels (or are related to different classes), PR finds the classes of the above-mentioned objects based on some specific algorithms. An example of PR could be to determine whether a given gravity profile is created by a spherical or non-spherical gravity source. PR has different types according to the kind of learning procedure used to define the classes of the objects. Learning procedures consist of supervised learning and unsupervised learning approaches. In the supervised learning approach, the training data should be available, consisting of some objects that have been properly classified. In the unsupervised learning approach, training data are not available and the procedure tries to find some patterns in the data that can be used to determine different kinds of input values. SVC is considered as a supervised learning algorithm of PR (Heijden et al. 2004). In gravity, producing training data is possible and therefore it is advisable to use a supervised learning algorithm. While producing the training data in gravity, it should be noted that we need to create several synthetic gravity profiles caused by synthetic gravity sources with different definite shapes. In this research, a set of synthetic gravity data caused by definite synthetic source shapes such as sphere, vertical cylinder, horizontal cylinder, anticline, syncline, and rectangular prism are prepared and considered as properly classified input values (training set). Then values of proper features from each of synthetic gravity profiles are extracted (see Sections 2.2 and 3). These values are used to train the supervised learning algorithm (SVC) and this trained algorithm would be able to estimate the shapes of sources of other gravity profiles including the real ones; as shown in Section 4.2, some real gravity sources have been estimated by SVC. For example, the shape of a well (a real gravity source) is estimated by the trained SVC with a shape of vertical cylinder. Estimation of gravity source shapes is a classification in which each gravity source shape is a class. 
Between different PR algorithms for gravity interpretation and other geophysical applications, unlike the SVC, neural network has been used frequently (Gret and Klingele 1998, Baan and Jutten 2000, Osman et al. 2006). Before this research, SVC has been used for depth estimation of faults (Hekmatian et al. 2013). However, according to our knowledge, SVC has not been used up to now for estimation of approximate shape of gravity sources. SVC algorithm has very suitable properties, such as a single global solution and ability to use different kernels. In this research we have shown that SVC algorithm can be adopted as a suitable method for estimating the approximate shapes of gravity sources.

\section{THEORIES AND ALGORITHMS}

\subsection{Support vector classifier (SVC) algorithm}

\subsubsection{Linear $S V C$}

Prior to linear SVC description we should note that support vector machine (SVM) is a concept in computer science for a set of related supervised learning methods that analyse data and recognize patterns used for classification and regression analysis; the original SVM formulations for classification is named SVC. SVC is clarified briefly in this and next sections, according to Heijden et al. (2004).

Linear (and non-linear) SVC considers only 2 classes of information that can be separated by a linear border. One class is located on one side of the linear border and the other is located on the other side of the linear border. If there are more than 2 classes, all classes except of one should be considered as one class and the remaining one as the other class. For example, we can consider the spherical shape of the gravity source as one class and all other possible shapes of the gravity source as another class. We should keep in mind that in SVC the border between the two classes is not a line but a margin and this border line is in the centre of the margin. The linear discriminant function that is used in linear SVC is (Heijden et al. 2004):

$$
\mathbf{g}\left(\mathbf{Z}_{\mathbf{n}}\right)-\mathbf{w}^{*} \mathbf{Z}_{\mathbf{n}}+b,
$$

where $\mathbf{Z}_{\mathbf{n}}$ is the matrix of the values of features (measurement vectors), $\mathbf{w}$ is the vector of coefficient, and $b$ is a scalar value. The rows of $\mathbf{Z}_{\mathbf{n}}$ are the features of each object (each gravity profile) and the columns of this matrix show the kind of features in all objects. Two classes $\left(C_{n}=1\right.$ and $C_{n}=-1$ are labels of two classes) are defined by Heijden et al. (2004) as:

$$
\mathbf{w}^{\mathrm{T}} \mathbf{Z}_{\mathbf{n}}+b \geq 1 \quad \text { if } C_{n}=+1,
$$

For all $n$

$$
\mathbf{w}^{\mathrm{T}} \mathbf{Z}_{\mathbf{n}}+b \leq-1 \quad \text { if } \quad C_{n}=-1,
$$




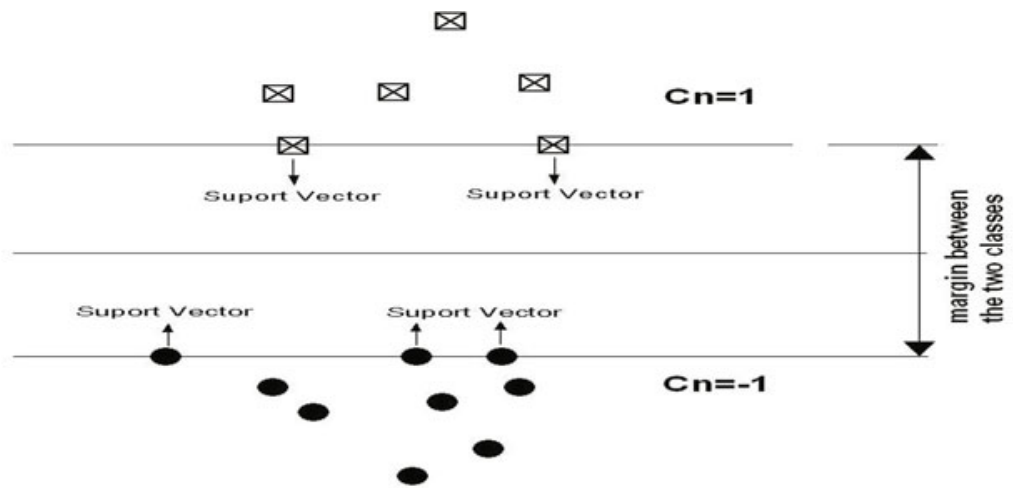

Fig. 1. The linear support vector classifier. The boundary between 2 classes is not only a margin but also a margin with maximum width and this shows the single global solution of SVC.

where $\mathbf{w}^{\mathbf{T}}$ means transpose of $\mathbf{w}$. The length of the margin between these two classes is shown in Fig. 1.

For more explanations regarding linear SVC, one of proper references is Heijden et al. (2004).

\subsubsection{Non linear $S V C$}

At first, some definitions should be recalled. The decision function is a function that maps the measurements space (features space) onto the set of possible classes. It should be emphasized that in SVC only 2 classes exist. Therefore, in linear and non-linear SVC, decision function consists of only 2 discriminant functions, $\mathbf{g}(\mathbf{Z}) \geq 1$ and $\mathbf{g}(\mathbf{Z}) \leq-1$; see Eqs. 1-3.

To have non-linear boundaries in SVC, the discriminant function should be defined as (Heijden et al. 2004):

$$
\mathbf{g}(\mathbf{Z})=\mathbf{w}^{\mathrm{T}} \mathbf{y}(\mathbf{Z})=\sum_{n=1}^{N_{s}} \mathbf{k}\left(\mathbf{Z}, \mathbf{Z}_{\mathbf{n}}\right),
$$

where $\mathbf{k}\left(\mathbf{Z}, \mathbf{Z}_{\mathbf{n}}\right)$ is the kernel (such as polynomial one), and $N_{s}$ is the number of samples in the training set. In Equation $4, \mathbf{g}(\mathbf{Z})=1$ and $\mathbf{g}(\mathbf{Z})=-1$ show the upper and lower limits of the margin of non-linear SVC, respectively (see Fig. 1 for comparing with linear SVC). Gaussian kernel could be used to develop a non-linear SVC and it can have a weighting matrix equal to $\sigma^{2} \mathbf{I}$ (I is the unit matrix). In this case, we have radial basis function kernel (RBF kernel) that has been applied in this study (Heijden et al. 2004):

$$
\mathbf{k}\left(\mathbf{Z}, \mathbf{Z}_{\mathbf{n}}\right)=\exp \left(-\frac{\left\|\mathbf{Z}-\mathbf{Z}_{\mathbf{n}}\right\|^{2}}{\sigma^{2}}\right),
$$


where $\sigma^{2}$ is a parameter for decreasing or increasing the power of exponential function of Eq. 5 .

\subsection{The features and feature selection (FS) algorithm}

Feature is a kind of measurement value which is obtained from each object. In this paper, object is a gravity profile which is created by a specific gravity source. Features that can be used for defining the shape of the gravity sources are derivable from a principal gravity profile. Gret and Klingele (1998) noted that the principal gravity profile is the gravity profile passing through the maximum value along the anomaly and crossing the anomaly lines perpendicularly. Gravity profiles in this research mean principal gravity profiles.

Some examples of the different features used to characterize the gravity anomalies are defined as (Gret and Klingele 1998):

$$
\begin{aligned}
& F 1=X_{g 50} / X_{g 75}, \\
& F 2=X_{g 75} / X_{g 50}, \\
& F 3=X_{g \text { inf }} / X_{g 75}, \\
& F 4=\left(X_{g 50}-X_{g 66}\right) /\left(X_{g 80}-X_{g 66}\right),
\end{aligned}
$$

where $g y$ is the value of gravity $(g)$ at $y \%$ of maximum value of gravity $g_{\max }$ and $X_{g y}$ is the value of $X$ at $g y$ (to be more specific, in our research $X$ is the horizontal distance in the direction of the profile from the location of $g_{\max }$ in the profile). Also, $X_{g}$ inf is the value of $X$ at $g$ inf and $g$ inf is the value of gravity $(g)$ at inflection point of the gravity profile. In Figure 2 an example of a

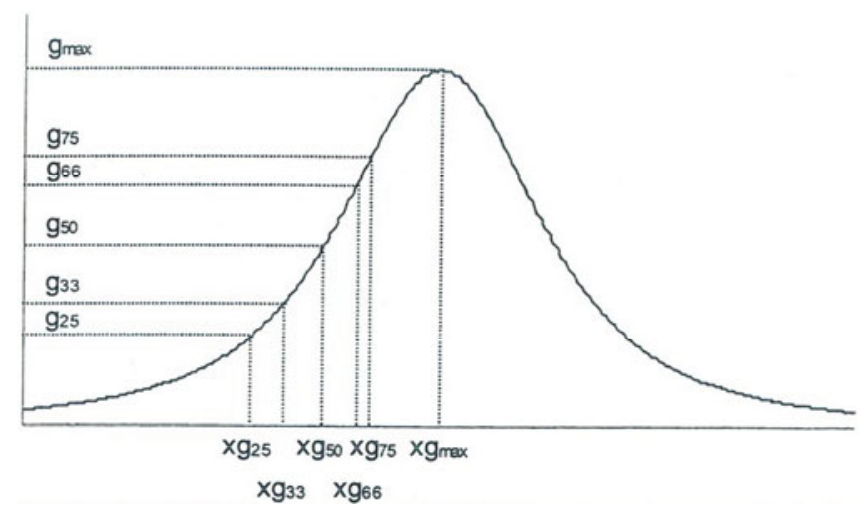

Fig. 2. Some of the parameters of features describing the shape of gravity sources. In this figure, $g y$ is the value of gravity $(g)$ at $y \%$ of maximum value of gravity $g_{\max }$ and $X_{g y}$ is the value of $X$ at $g y$ (Gret and Klingele 1998). 
gravity profile and some of the parameters of the features describing the shape of gravity sources are shown.

Choosing proper features (feature selection, FS) is very important while dealing with high misclassification in testing our classifier (more proper features are those features which, when used in classification, lead to better separation of the classes). The high misclassification in our selected features space means that the different classes are not separated properly. One of the enable FS algorithms is "inter/intra class distance" (IICD) and we have used this algorithm in our research. The IICD algorithm, like all other FS algorithms, has a criterion for selection of more proper features. This criterion is called performance measure (PM). PM increases with increasing suitability of the features for classification, and vice versa; in other words, the selection of more proper features is done when their PM is bigger. PM of IICD is based on Euclidean distance between each of the two objects in the training set (all objects used for training SVC) in the features space. For more explanation about "inter/intra class distance" (IICD) algorithm, we should mention that, as Heijden et al. (2004) noted, $T_{s}$ is a training set with $N_{s}$ samples (objects). The classes $w_{k}$ are represented by subsets $T_{k}$ (subsets $T_{k}$ are subsets of objects of $T_{s}$ ), each class having $N_{k}$ samples. Measurement vectors in $T_{s}$ without reference to their class are denoted by $\mathbf{Z}_{\mathbf{n}}$ (For each object we measure a set of features. This set of features is called measurement vector. The point location of each object in features space is defined with its measurement vector.). Measurement vectors in $T_{k}$ (i.e., vectors coming from class $w_{k}$ ) are denoted by $\mathbf{Z}_{\mathbf{k}, \mathbf{n}}$. The sample mean of class $w_{k}$ is (Heijden et al. 2004):

$$
\hat{\mu}_{k}=\frac{1}{N_{k}} \sum_{n=1}^{N_{k}} \mathbf{Z}_{\mathbf{k}, \mathbf{n}} .
$$

The sample mean of the entire training set is (Heijden et al. 2004):

$$
\hat{\mu}_{k}=\frac{1}{N_{s}} \sum_{n=1}^{N_{s}} \mathbf{Z}_{\mathbf{n}} .
$$

We can represent the distances between objects or samples (of the training set) by means of scatter matrices. A scatter matrix gives some information about the dispersion of a population of samples around their mean. For instance, the matrix that describes the scattering of vectors from class $w_{k}$ is (Heijden et al. 2004):

$$
\mathbf{S}_{\mathbf{k}}=\frac{1}{N_{k}} \sum_{n=1}^{N_{k}}\left(\mathbf{Z}_{\mathbf{k}, \mathbf{n}}-\hat{\mu}_{k}\right)\left(\mathbf{Z}_{\mathbf{k}, \mathbf{n}}-\hat{\mu}_{k}\right)^{\mathbf{T}},
$$

where $\mathbf{S}_{\mathbf{k}}$ supplies information about the average distance of the scattering in class $w_{k}$. Averaged over all classes, the scatter matrix is (Heijden et al. 2004): 


$$
\mathbf{S}_{\mathbf{w}}=\frac{1}{N_{s}} \sum_{k=1}^{K} N_{k} \mathbf{S}_{\mathbf{k}}=\frac{1}{N_{s}} \sum_{k=1}^{K} \sum_{n=1}^{N_{k}}\left(\mathbf{Z}_{\mathbf{k}, \mathbf{n}}-\hat{\mu}_{k}\right)\left(\mathbf{Z}_{\mathbf{k}, \mathbf{n}}-\hat{\mu}_{k}\right)^{\mathbf{T}} .
$$

This matrix is "the within-scatter matrix", as it describes the average scattering within classes. In addition, there is "the between-scatter matrix", $\mathbf{S}_{\mathbf{b}}$, that describes the scattering of the class-dependent sample means around the overall average (Heijden et al. 2004):

$$
\mathbf{S}_{\mathbf{b}}=\frac{1}{N_{s}} \sum_{k=1}^{K} N_{k}\left(\hat{\mu}_{k}-\hat{\mu}\right)\left(\hat{\mu}_{k}-\hat{\mu}\right)^{\mathbf{T}} .
$$

We know that an individual number is a kind of matrix. So, when "the within-scatter matrix" $\left(\mathbf{S}_{\mathbf{w}}\right)$ is an individual number, it shows the squared average distance of the location of each object in one class from the average location of all objects of that class in features' space for all the classes. In this case, we name $\mathbf{S}_{\mathbf{w}}$ as intraclass distance, and when "the between-scatter matrix" $\left(\mathbf{S}_{\mathbf{b}}\right)$ is an individual number, it refers to the squared average distance of the average location of all objects of each class from the average location of all objects of all classes in features space. In this case, we name $\mathbf{S}_{\mathbf{b}}$ as interclass distance. The features that are more suitable for classification are the ones for which their interclass distance is greater than their intraclass distance. The performance measure (PM) suited to express the separability of classes is the ratio between interclass and intraclass distance. Therefore, features with greater PM are preferred for classification (according to IICD algorithm).

Also we should take into consideration the fact that the number of possible subsets, $q(D)$, consisting of $D$ features between $N$ features is (Heijden et al. 2004):

$$
q(D)=\frac{N !}{(N-D) ! D !} .
$$

We have used the FS approach, as will be described in what follows.

\section{THE PROCEDURE}

In this procedure it was necessary to develop a software, which was done. For producing synthetic gravity profiles from different gravity sources and for extracting features from each gravity profile, a software package was produced using Visual Basic language. The algorithms of gravity calculation which we used for preparing the above-mentioned software were obtained from different references. The algorithm of gravity calculation with sources of spherical shape was obtained from Telford et al. (1976), and that of anticline or syncline shape from Talwani et al. (1959), with rectangular prism 
shape obtained from Plouff (1976), horizontal cylinder shape obtained from Blakely (1996) and vertical cylinder shape obtained from Belikov (1978). For features selecting (FS) we produced 3 softwares using Visual Basic language. The algorithms of FS which we used for preparing our softwares were obtained from Heijden et al. (2004). These softwares are, respectively, able to select more suitable 2, 3, and 4 features out of 10 features. Also, we produced and trained tens of SVC codes for estimation of the gravity sources shapes. For producing the codes we used Matlab environment and also some tools of PRTools 4.1 and its manual (Duin et al. 2007) which can be found in website http://www.prtools.org/.

The procedure of this study includes 6 steps as follows:

(1) Three hundred synthetic gravity profiles (training set) were created by gravity sources with 6 definite shapes and different dimensions, depths and density contrasts for training SVC. For each of the 6 shapes, 50 synthetic gravity profiles were created. The specifications of the sources of these 300 synthetic gravity profiles are presented in Table 1.

Table 1

Gravity sources with different shapes, dimensions, depths, and density contrasts used for creating synthetic gravity profiles (to be used for training set)

\begin{tabular}{|c|c|c|c|c|c|}
\hline Shapes & $\begin{array}{c}\text { Radius } \\
{[\mathrm{m}]}\end{array}$ & $\begin{array}{l}\text { Dimensions } \\
\text { except radius } \\
{[\mathrm{m}]}\end{array}$ & $\begin{array}{c}\text { Minimum } \\
\text { depth } \\
{[\mathrm{m}]}\end{array}$ & $\begin{array}{c}\text { Maximum } \\
\text { depth } \\
{[\mathrm{m}]}\end{array}$ & $\begin{array}{c}\text { Density } \\
\text { contrast } \\
{[\mathrm{gr} / \mathrm{cc}]}\end{array}$ \\
\hline Sphere & 6 to 365 & & 5 to 600 & & 0.5 to 1.5 \\
\hline $\begin{array}{l}\text { Horizontal } \\
\text { cylinder }\end{array}$ & 5 to 240 & & 5 to 600 & & 0.5 to 1.2 \\
\hline $\begin{array}{l}\text { Vertical } \\
\text { cylinder }\end{array}$ & 5 to 500 & $\begin{array}{l}5 \text { to } 100 \\
\text { (height) }\end{array}$ & 5 to 500 & & 0.5 to 1.1 \\
\hline $\begin{array}{l}\text { Rectangular } \\
\text { prism }\end{array}$ & & $\begin{array}{c}6 \text { to } 1100 \\
\text { (in direction of the profile) } \\
2 \text { to } 100 \\
\text { (perpendicular to the profile) }\end{array}$ & 3 to 600 & 5 to 700 & 0.4 to 1.2 \\
\hline Anticline & & $\begin{array}{c}4 \text { to } 185 \\
\text { (vertical extension) } \\
20 \text { to } 400 \\
\text { (horizontal extension) }\end{array}$ & 5 to 500 & & 1.0 \\
\hline Syncline & & $\begin{array}{c}4 \text { to } 185 \\
\text { (vertical extension) } \\
20 \text { to } 400 \\
\text { (horizontal extension) }\end{array}$ & 5 to 500 & & 1.0 \\
\hline
\end{tabular}


(2) We extracted 10 features from each of the above-mentioned 300 synthetic gravity profiles. These ten features are shown in Table 2.

Table 2

The extracted features

\begin{tabular}{|c|c|}
\hline $\begin{array}{c}\text { Number } \\
\text { of the extracted features }\end{array}$ & Extracted features \\
\hline 1 & $\left(X_{g 50} / X_{g 80}\right)$ \\
2 & $\left(X_{60} / X_{g 80}\right)$, \\
3 & $\left(X_{\text {ginf }} / X_{g 80}\right)$ \\
4 & $\left(X_{g 50} / X_{g 70}\right)$ \\
5 & $\left(X_{g 60} / X_{g 70}\right)$ \\
6 & $\left(X_{g \text { inf }} / X_{g 70}\right)$ \\
7 & $\left(X_{g 50} / X_{g 90}\right)$ \\
8 & $\left(X_{g 60} / X_{g 90}\right)$ \\
9 & $\left(X_{g i n f} / X_{g 90}\right)$ \\
10 & $\left(\left(X_{g 50}-X_{g 80}\right) / X_{g 90}\right)$ \\
\hline
\end{tabular}

Note: All the parameters are introduced in Section 2.2.

As shown in Table 2, we specified each of the extracted features with a number from 1 to 10. By having these ten features from each of the profiles, we would be able to select more suitable features for classification according to IICD algorithm (see Section 2.2). We should mention that these 10 features are good references for FS because they are related to the shape of the gravity sources and are independent of the depths and dimensions of the sources and also consist of all the $X_{g y}$ from $X_{g 50}$ to $X_{g 90}$, systematically.

(3) We selected more properly 2,3 , or 4 features out of 10 . According to Eq. 15, the possible numbers of 2, 3, or 4 features (subsets) are 45, 120, and 210 , respectively. But from these 45, 120, and 210 subsets (a total of 375 subsets), respectively, only 10,17 , and 14 have relatively large performance measures (see Section 2). By the values of the features of these 10, 17, and 14 subsets we trained, respectively, 10,17, and 14 SVC codes for classification or, better to say, for estimation of the gravity sources shapes.

(4) The best trained SVC codes were related to only one subset of 3 features (includes $\left(X_{g 50} / X_{g 80}\right),\left(X_{g i n f} / X_{g 80}\right)$, and $\left(\left(X_{g 50}-X_{g 80}\right) / X_{g 90}\right)$, i.e., features 1,3 , and 10 regarding to Table 2 ) that was able to classify more than 0.70 of objects in the training set correctly (therefore, the best subset of features is the subset not only with big PM but also should classify more objects correctly than the other subsets of features). So we tested this best trained SVC with 60 more synthetic gravity profiles (testing set). The above- 
mentioned testing set consists of 10 profiles for each of the 6 shapes which we want to classify. The best trained SVC codes were able to classify the testing set properly (so that the best trained SVC code is the one that is able to classify the testing set most properly). But for more accurate estimation, we decided to train more SVC codes with a different approach (explained in the next step). Before going to the next step, let us make two comments. First, it should be mentioned that in testing all the trained SVCs, we inserted, as a test, some noises in some of gravity profiles with different values equal to about one twentieth of the average value of the gravity profiles. These noises caused a little effect on the values of the extracted features (and so caused a little effect on the success of classification) except on those features (of Table 2) that consist of $X_{g \text { inf }}$ (see Section 2.2). This is so because $X_{g \text { inf }}$ is the value of $X$ at $g$ inf and $g$ inf is the value of gravity $(g)$ at inflection point of the profile and, by inserting noise, new inflection points will be created and it will become too hard to locate the principal inflection point in this situation. The second comment is related to the learning procedure. For learning SVC, it is needed to create a training dataset consisting of the features of each object in the dataset and a number (1 or 2) indicating the class of each object (SVC is considering 2 classes only). Then SVC (with the kernel (RBF) and its parameter $\sigma$ (see Eq. 5) will use the training dataset to designate the boundary between the two classes. By trails and errors, it is found that the proper value for the parameter $\sigma$ is 0.05 . With RBF kernel with the above-mentioned value for its parameter, the misclassification was (with respect to other kernels and parameters) low. The SVC code with this kernel and the best subset of features can be assumed as the best SVC code.

(5) For better estimation of gravity sources shapes, we introduced 4 classification groups, such that each group consists of two classes and each class consists of three shapes. These 4 groups are shown in Table 3.

In other words, for better classification, the six shapes are classified by four classification groups and each of these 4 classification groups consists of two classes, while each class consists of three shapes. These four groups and related information are shown in Table 3. For each of the classification groups we used a couple of codes. For example, according to Table 3, for the first classification group one SVC code is trained to separate Elongated shapes from Non-Elongated shapes and one other SVC code trained to separate Limited shapes from Non-Limited shapes. These two codes do the same duty (separating Elongated shapes from Limited shapes) but one of these two codes tries to detect Limited shapes (those objects that are not detected as Limited shapes will be introduced as Non-Limited shapes) and the other code does the same duty for Elongated shapes. If we use all these 4 couples of codes, we will be able to classify all the 6 gravity sources shapes, because each of the 6 shapes has different responses according to all the 4 couples of 
Four classification groups of 2 three shapes classes

\begin{tabular}{|c|l|l|l|l|}
\hline $\begin{array}{c}\text { Number } \\
\text { of group }\end{array}$ & \multicolumn{1}{|c|}{$\begin{array}{c}\text { Name } \\
\text { of class 1 }\end{array}$} & $\begin{array}{c}\text { The related gravity } \\
\text { sources shapes } \\
\text { of class 1 }\end{array}$ & $\begin{array}{c}\text { Name } \\
\text { of class 2 }\end{array}$ & $\begin{array}{c}\text { The related gravity } \\
\text { sources shapes } \\
\text { of class 2 }\end{array}$ \\
\hline 1 & $\begin{array}{l}\text { Elongated } \\
\text { shapes }\end{array}$ & $\begin{array}{l}\text { Horizontal cylinder, } \\
\text { Anticline and } \\
\text { Syncline }\end{array}$ & $\begin{array}{l}\text { Limited } \\
\text { shapes }\end{array}$ & $\begin{array}{l}\text { Vertical cylinder, } \\
\text { Rectangular prism } \\
\text { and Sphere }\end{array}$ \\
\hline 2 & Up curved & $\begin{array}{l}\text { Horizontal cylinder, } \\
\text { Anticline and Sphere }\end{array}$ & Up Flat & $\begin{array}{l}\text { Vertical cylinder, } \\
\text { Rectangular prism } \\
\text { and Syncline }\end{array}$ \\
\hline 3 & Down curved & $\begin{array}{l}\text { Horizontal cylinder, } \\
\text { Syncline and Sphere }\end{array}$ & Down Flat & $\begin{array}{l}\text { Vertical cylinder, } \\
\text { Rectangular prism } \\
\text { and Anticline }\end{array}$ \\
\hline 4 & Circle section & $\begin{array}{l}\text { Horizontal cylinder, } \\
\text { Vertical cylinder } \\
\text { and Sphere }\end{array}$ & $\begin{array}{l}\text { Non Circle } \\
\text { section }\end{array}$ & $\begin{array}{l}\text { Rectangular prism, } \\
\text { Anticline and } \\
\text { Syncline }\end{array}$ \\
\hline
\end{tabular}

codes. For example, horizontal cylinder based on the codes related to Group 1 through Group 4 is classified, respectively, as: Elongated shapes, Up curved, Down curved, and Circle section. Likewise, anticline is classified, respectively, as: Elongated shapes, Up curved, Down flat, and Non Circle section. In this way, all the 6 shapes have different classes regarding all the four groups. Therefore, only by using these 4 couples of codes we are able to classify all the 6 shapes of the gravity sources. By using these 4 couples of codes, most of the 60 synthetic gravity profiles that were used for testing were classified correctly and testings with real gravity profiles were good. Considering Table 3, the six possible shapes of gravity sources in our research have the classes as presented in Table 4.

On the other hand, using all these 4 couples of codes will give us opportunity to confirm or reject the result of each other. For example, if the result of one of these 4 couples of codes is Elongated shape and results of 2 others are Up flat and Down flat, we will understand that the gravity profile is not interpretable by these 4 couple of codes. The reason is that none of the 6 shapes have these 3 properties (Elongated shape, Up flat, Down flat) simultaneously (see Table 4.). In such conditions, the gravity profile may not be a principal one (see Section 2.2).

(6) Although the above-mentioned codes until now have been sufficient for classifying the 6 shapes, for classifying with greater certainty, we trained more SVC codes. Based on Table 3 we have 8 classes and each class consists of 3 shapes. Thus, we trained twenty four $(8 \times 3=24)$ other codes so 
The classes of the 6 possible shapes of gravity sources in our research based on Table 3

\begin{tabular}{|l|l|}
\hline \multicolumn{1}{|c|}{ The shape of the gravity source } & \multicolumn{1}{c|}{ The related classes } \\
\hline Anticline & $\begin{array}{l}\text { Elongated shape, Up curved, Down } \\
\text { flat, Non Circle section }\end{array}$ \\
\hline Horizontal cylinder & $\begin{array}{l}\text { Elongated shape, Up curved, Down } \\
\text { curved, Circle section }\end{array}$ \\
\hline Rectangular prism & $\begin{array}{l}\text { Limited shape, Up flat, } \\
\text { Down flat, Non Circle section }\end{array}$ \\
\hline Sphere & $\begin{array}{l}\text { Limited shape, Up curved, } \\
\text { Down curved, Circle section }\end{array}$ \\
\hline Syncline & $\begin{array}{l}\text { Elongated shape, Up flat, } \\
\text { Down curved, Non Circle section }\end{array}$ \\
\hline Vertical cylinder & $\begin{array}{l}\text { Limited shape, Up flat, } \\
\text { Down flat, Circle section }\end{array}$ \\
\hline
\end{tabular}

that each of them can estimate the shape out of 3 shapes. For more explanations see the next section.

Finally, it should be noted that, in this paper, the SVC has not only been used for estimating the approximate shape of the gravity sources, but we also modified our approach by training different SVC codes. And justification of this approach is that these different SVC codes can test the result of each other.

The flowchart of the above-mentioned 6 steps is as follows:

(1) 300 synthetic gravity profiles (training set) were created by gravity sources with definite shapes (6 shapes), dimensions, depths, and density contrasts for training SVC.

(2) We extracted 10 features from each of the 300 synthetic gravity profiles.

(3) Regarding our FS procedures:

(a) We selected the more proper 2, 3, and 4 features out of the 10 features based on IICD algorithm.

(b) We trained SVC codes (using different proper subsets) and tested them by the training set. We selected the best trained SVC codes (in which we use the best subset of features). 
(4) We tested the best trained SVC codes with 60 more synthetic gravity profiles different from the training set. The results were relatively good but for better classification we modify our approach in the next step.

(5) We trained SVC codes for estimating the 6 shapes in 4 classification groups of 2 three shapes classes (in which we used the best subset of features). And the results of testing were better.

(6) For classifying with greater certainty we trained 24 more SVC codes (in which we used the best subset of features). Each of these 24 codes can estimate the shape between 3 shapes.

\section{RESULTS AND DISCUSSIONS}

\subsection{Synthetic data (and some other discussions)}

The results of testing with the training set showed us that using 2-feature subsets for our classification is not suitable. Examining the trained SVC codes related to 17 proper 3 -feature subsets (see point 3 in Section 3) with the training set showed that only one of them is able to classify more than 0.70 of the objects in the training set correctly and we save this subset for more testing. Finally, testing the trained SVC codes related to the 14 proper 4 -feature subsets with the training set showed us that they are able (with their best subset) to classify only 0.60 of objects in the training set correctly. Therefore, for classification we returned to only one subset of 3 features that was able to classify more than 0.70 of the objects in the training set correctly. Before discussing the above-mentioned best or most suitable subset of 3 features, in the following we show some results of using 2-feature subsets.

In the best case of testing with the training set using 2-feature subsets, only 0.42 of 50 anticlines in the training set are classified correctly (as shown in Fig. 3).

The results illustrate that using only 2 features for our classification is not suitable. So we do not continue discussion about using only 2 features for our classification.

As mentioned in point 3 of Section 3, according to Eq. 15, the possible number of 3 -features subsets is 120 . But from these 120 subsets only 17 have relatively big performance measures (see Section 2.2). These 17 subsets are the more proper subsets of features and these are shown in Table 5.

As we mentioned earlier, the best subset of features for classification (estimation) of the gravity source shape is one of the 3-feature subsets. As it was mentioned in point 4 of Section 3 , this subset includes $\left(X_{g 50} / X_{g 80}\right)$, 


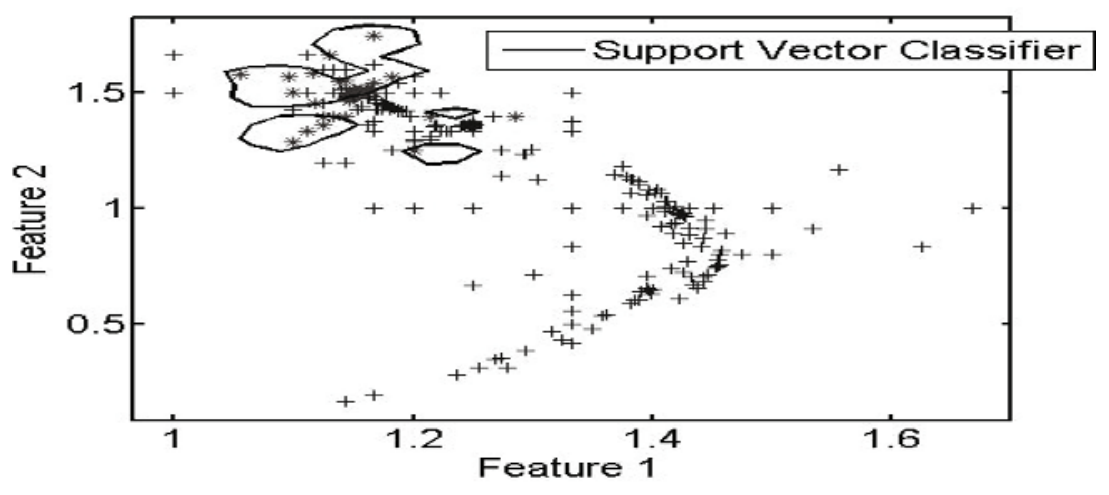

Fig. 3. The border of one of the trained SVC (related to the best 2-feature subset) and points showing the features of 300 training synthetic gravity profiles. This figure shows the trained SVC for detecting anticline (star symbols) and non-anticline (cross symbols) gravity source shape in 2-dimensional features space.

Table 5

17 subset of 3 features (between 120 possible subsets) with relatively big performance measures (PM)

\begin{tabular}{|c|c|c|c|}
\hline $\begin{array}{c}\text { Feature 1 } \\
\text { number }\end{array}$ & $\begin{array}{c}\text { Feature 2 } \\
\text { number }\end{array}$ & $\begin{array}{c}\text { Feature 3 } \\
\text { number }\end{array}$ & PM \\
\hline 5 & 6 & 10 & 3.397 \\
3 & 6 & 10 & 3.394 \\
4 & 6 & 10 & 3.371 \\
1 & 6 & 10 & 3.358 \\
2 & 6 & 10 & 3.180 \\
1 & 3 & 10 & 3.169 \\
1 & 5 & 10 & 3.156 \\
1 & 4 & 10 & 3.150 \\
3 & 5 & 10 & 3.148 \\
1 & 3 & 6 & 3.143 \\
3 & 4 & 10 & 3.141 \\
4 & 5 & 10 & 3.123 \\
1 & 5 & 6 & 3.106 \\
1 & 4 & 6 & 3.098 \\
3 & 4 & 6 & 3.036 \\
3 & 5 & 6 & 3.033 \\
1 & 2 & 10 & 3.008 \\
\hline
\end{tabular}

Note: Regarding feature numbers, see Table 2. 
$\left(X_{g \text { inf }} / X_{g 80}\right)$ and $\left(\left(X_{g 50}-X_{g 80}\right) / X_{g 90}\right)$, which are features 1, 3, and 10, regarding Table 2. In Table 6 we present the portion of each of the 6 gravity sources shapes which classified correctly using the best subset of features and using the related trained SVC codes.

Using the best 3 -feature subset, 0.74 of 50 horizontal cylinders in the training set are classified correctly and are presented in Fig. 4.

As stated earlier about non-linear SVC, the kernel which used is RBF kernel (Eq. 5). The value of $\sigma$ in the kernel is selected to be equal to 0.05 .

As mentioned in point 5 of Section 3, for better classification, the six shapes are classified by four classification groups and each of these 4 classification groups consists of two classes, each class consisting of three shapes.

Table 6

The portion of each of the 6 gravity source shapes (in the training and testing set) which classified correctly using the most suitable subset of features and using the related trained codes

\begin{tabular}{|l|c|c|}
\hline \multicolumn{1}{|c|}{$\begin{array}{c}\text { The shape } \\
\text { of gravity source }\end{array}$} & $\begin{array}{c}\text { The portion of the shape } \\
\text { (in the training set) } \\
\text { which classified correctly }\end{array}$ & $\begin{array}{c}\text { The portion of the shape } \\
\text { (in the testing set) } \\
\text { which classified correctly }\end{array}$ \\
\hline Anticline & $(36 / 50)=0.72$ & $(5 / 10)=0.5$ \\
Horizontal cylinder & $(37 / 50)=0.74$ & $(5 / 10)=0.5$ \\
Rectangular prism & $(50 / 50)=1$ & $(3 / 10)=0.3$ \\
Sphere & $(49 / 50)=0.98$ & $(10 / 10)=1.0$ \\
Syncline & $(48 / 50)=0.96$ & $(5 / 10)=0.5$ \\
Vertical cylinder & $(49 / 50)=0.98$ & $(6 / 10)=0.6$ \\
\hline
\end{tabular}

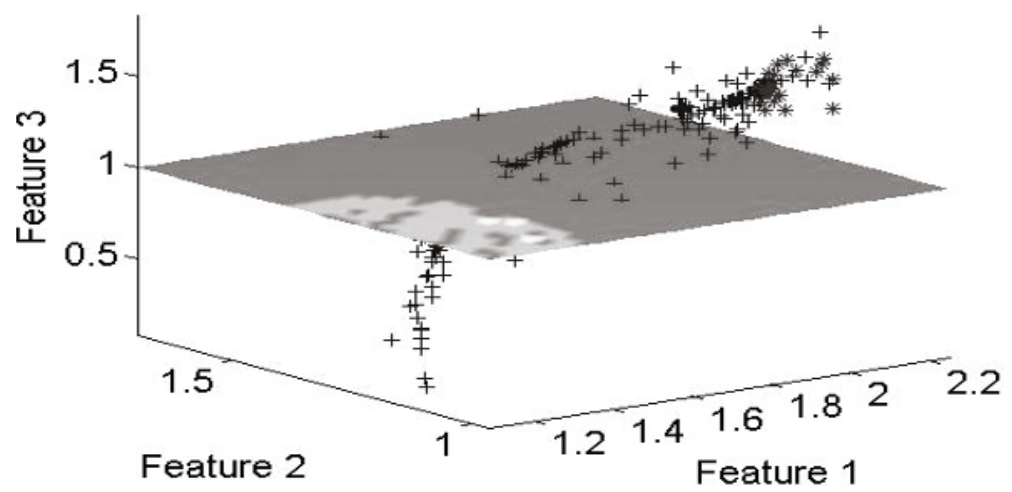

Fig. 4. One of the trained SVC (related to the best 3-feature subset) and points showing the features of 300 training synthetic gravity profiles. This figure shows the trained SVC for detecting horizontal cylinder (star symbol) and non-horizontal cylinder (cross symbol) gravity source shape in 3-dimensional features space. 
These four groups and related information are shown in Table 3. For each of the 4 above-mentioned classification groups we used a couple of codes. The results of testing of one of these 4 couples of codes by training set and testing set are shown in Table 7.

Table 7

The portion of each of the 6 gravity sources shapes (in the training and testing set) which classified correctly using the most suitable subset of features and using one of the 4 couples of codes

\begin{tabular}{|l|c|c|}
\hline \multicolumn{1}{|c|}{$\begin{array}{c}\text { The shape } \\
\text { of gravity source }\end{array}$} & $\begin{array}{c}\text { The portion of the shape } \\
\text { (in the training set) } \\
\text { which classified correctly }\end{array}$ & $\begin{array}{c}\text { The portion of the shape } \\
\text { (in the testing set) } \\
\text { which classified correctly }\end{array}$ \\
\hline Anticline & $(49 / 50)=0.98$ & $(9 / 10)=0.9$ \\
Horizontal cylinder & $(50 / 50)=1$ & $(10 / 10)=1.0$ \\
Rectangular prism & $(50 / 50)=1$ & $(7 / 10)=0.7$ \\
Sphere & $(49 / 50)=0.98$ & $(10 / 10)=1.0$ \\
Syncline & $(50 / 50)=1$ & $(5 / 10)=0.5$ \\
Vertical cylinder & $(50 / 50)=1$ & $(9 / 10)=0.9$ \\
\hline
\end{tabular}

The results of testing 3 others of the 4 couples of codes are suitable and almost all of the shapes were classified correctly.

As stated before, for each of the discussed 4 classification groups we have 2 classes. So all together we have eight classes, each of them consisting of 3 shapes. Therefore, for the six gravity sources shapes, eight groups of the three shapes (all together 24 shapes) exist. In this regard, we produced and trained 24 SVC codes (8 groups of codes, each consisting of 3 codes) so that each of these codes can separate one shape out of the three shapes. In Table 8 these eight groups of three shapes are represented.

Table 8

Names of the eight groups of the 3 shapes for which the related 24 codes can classify (estimate) the shape of the gravity source between 3 possible shapes using the most suitable subset of features

\begin{tabular}{|l|}
\hline \multicolumn{1}{|c|}{ Names of the eight of the 3 shapes } \\
\hline Anticline, Horizontal cylinder, Syncline \\
Rectangular Prism, Sphere, Vertical cylinder \\
Anticline, Horizontal cylinder, Sphere \\
Rectangular Prism, Syncline, Vertical cylinder \\
Horizontal cylinder, Sphere, Syncline \\
Anticline, Rectangular prism, Vertical cylinder \\
Horizontal cylinder, Sphere, Vertical cylinder \\
Anticline, Rectangular prism, Syncline \\
\hline
\end{tabular}


In the next section we have used some of these $24 \mathrm{SVC}$ codes in the shape estimation.

It should be mentioned that we created some type of SVC codes so that each of them individually is able to estimate the approximate shape of gravity source. But if we test each type of these codes with the others and if some or all of these types of SVC codes give us the same result (the estimate of the approximate shape of the gravity source is the same), we will be more assured about the estimated approximate shape of the gravity source than when we only use one type of our SVC codes for shape estimation. So logically the modified approach we introduced (using not only one but some or all of our different types of SVC codes for shape estimation) leads us to more liable results. In any way, the results of classifications of 2 types of our trained SVC codes are presented in Tables 6 and 7. So they can be compared.

Finally, we should say that, as we noted in the Introduction, there is no usual method and no unique result for estimation of initial assumption of shape of gravity source (approximate shape of gravity sources) and practically this assumption is prepared by non gravity data. So any trial in this regard is useful and therefore using SVC (especially with more proper features) will be a step towards better estimation of initial assumption of shapes of gravity sources.

\subsection{Real data}

In this Section we have used two sets of real data for testing our approach. The approximate location of these 2 sets of real data in Iran is shown in Fig. 5.

The first set of real data belongs to the small grid gravity network at the Institute of Geophysics of the University of Tehran. The aim was to detect and model the tunnels of an old buried channel. The grid space of $2 \times 2 \mathrm{~m}^{2}$ is used for measurements. In both, first (Fig. 6) and second (Fig. 7) sets of real data, a Scintrex (CG3M) gravimeter with 1 micro-gal resolution is used and the coordinates of the points are measured by the Total Station (Leika 750) with an accuracy to a few centimetres in the $x, y$, and $h$ coordinates. And also in both sets of real data, after gravity corrections due to the standard formulas, the Bouguer gravity anomalies are computed and, by removing the trend effect with polynomial fitting method, the residual anomalies are computed. The first set of real data is shown in Fig. 6.

A gravity profile (line AB in Fig. 6) is selected for testing. This profile was collected above a well, i.e., the gravity source of this gravity profile is a well filled with air. We knew that the well is very similar in shape to a vertical cylinder or a long vertical rectangular prism. 


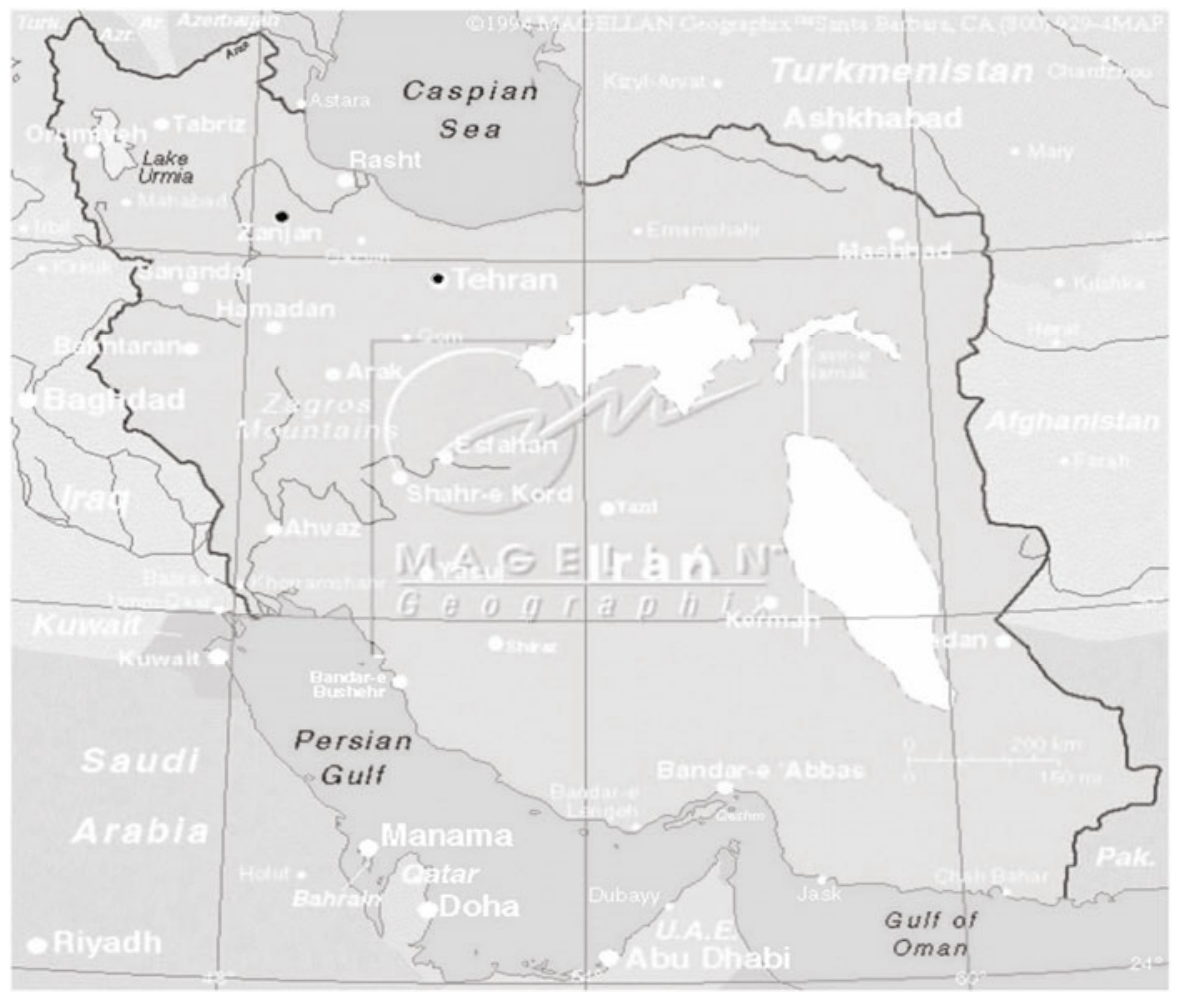

Fig. 5. Dark spots show the approximate locations of the 2 sets of real data in Iran. The geographic map of Iran is extracted from Ardestani (2008).

The second set of real data belongs to the grid gravity network carried out in a mining site close to Zanjan (a city in Iran) for searching for a manganese ore body. The grid space of $10 \times 10 \mathrm{~m}^{2}$ is used. This set of real data is shown in Fig. 7.

The three real gravity profiles which we used in this set of real data are presented in Fig. 7 as profiles CD, EF, and GH. The 3D inversion according to the approach of Camacho et al. (2002) was applied on window 2 (this window is the big rectangle that is around not only the 3 above-mentioned profiles but also other areas in Fig. 6) of the second set of gravity data and this inversion is shown in Fig. 8.

In the above-mentioned gravity inversion, the aim has been to detect density differences of the sources with background and to detect approximate locations and dimensions of the sources.

The results of executing the trained SVC codes related to the 4 classification groups on the above-mentioned real gravity profiles are shown in Table 9. 


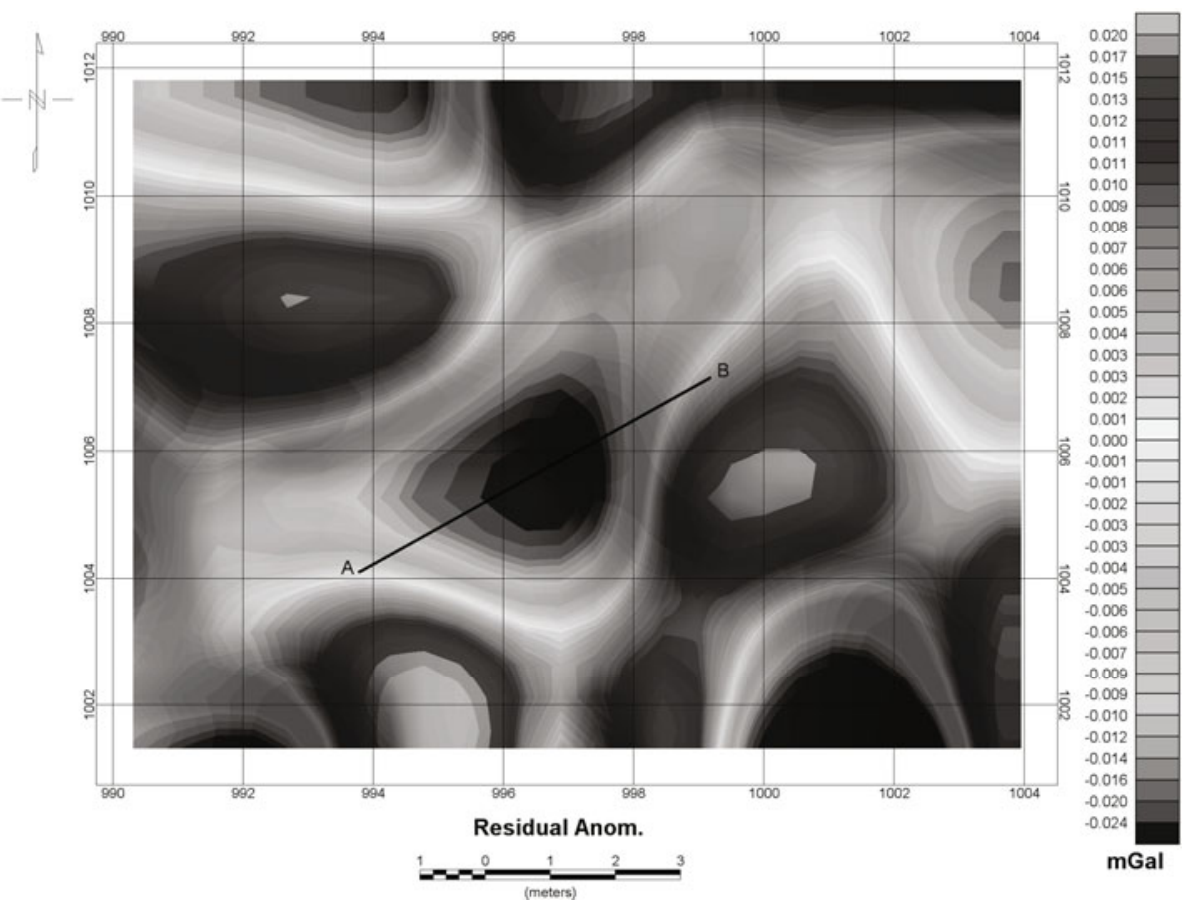

Fig. 6. Location of the gravity profile $\mathrm{AB}$ over the well. The map of this figure is a residual gravity map of a part of the Institute of Geophysics of University of Tehran and the coordinates are the local one (Ardestani 2009).

Regarding the calculation of probability of being of one specific class (for example, the result of executing one of the SVC codes on profile EF is that its source has Limited shape (a specific class) with probability 0.77 (as shown in Table 9)), we should refer to Fig. 1 for more explanations. In Figure 1, all the objects in the area above the line showing the upper limit of the margin are related to the class with the label $C_{n}=1$ with probability 1 $(100 \%)$, but if we have (in Fig. 1) any object between the line showing the upper limit of the margin and the line showing the middle of the margin, then this object would be related to the class with the label $C_{n}=1$ with a probability between 100 and $50 \%$. Also, if we have any object between the line showing the middle of the margin and the line showing the lower limit of the margin, then this object would be related to the class with the label $C_{n}=1$ with a probability between 50 and $0 \%$. And in areas lower than the line showing the lower limit of the margin, any object is related to the class with the label $C_{n}=1$ with a probability equal to $0 \%$. For all the objects, it should be mentioned that they are related to the class with the label $C_{n}=-1$ with probability equal to " 1 - probability of being related to the class with 


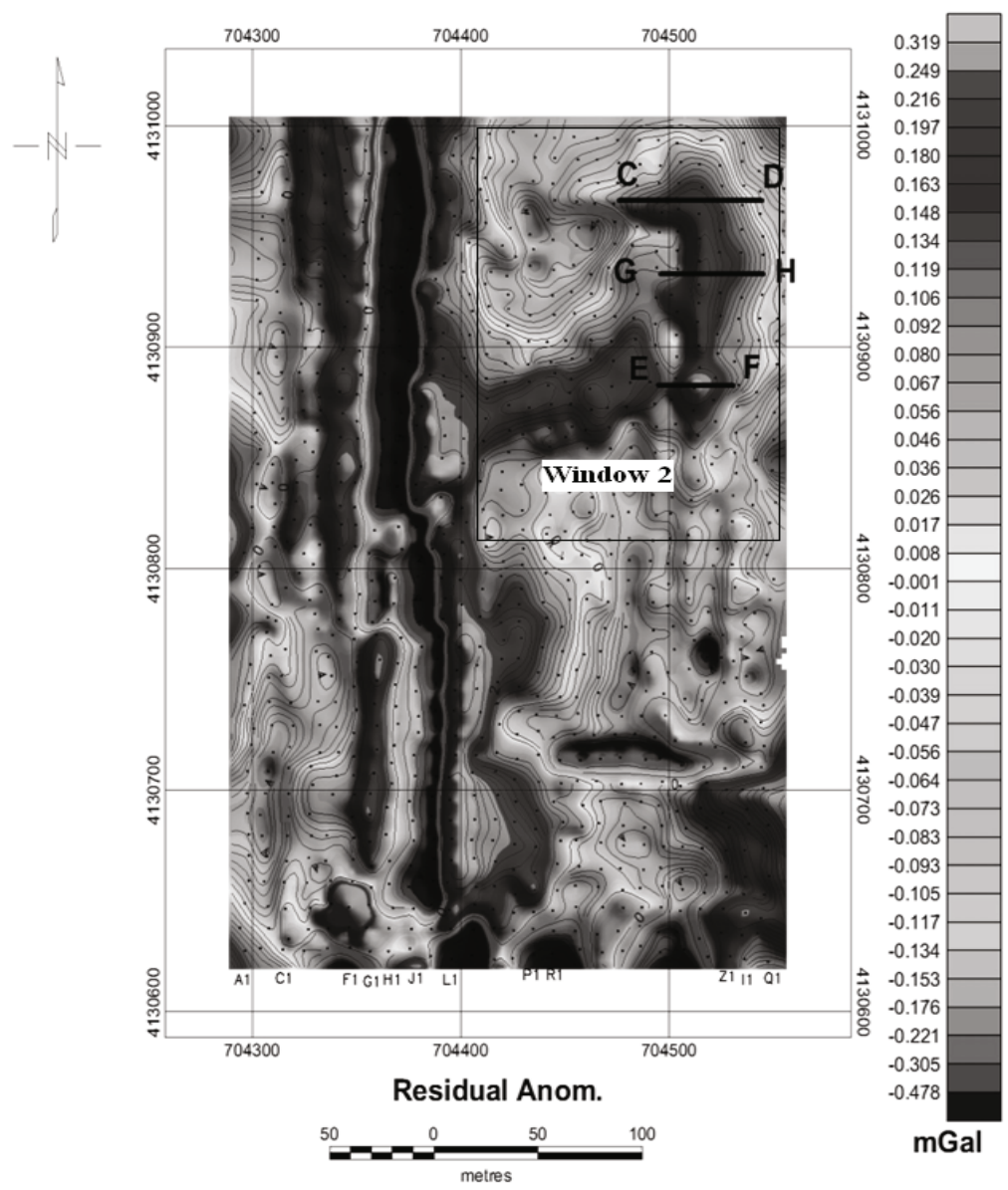

Fig. 7. Locations of the gravity profiles $\mathrm{CD}, \mathrm{EF}$, and GH. The map of this figure is a residual gravity map of a mining site near Zanjan (a city in Iran) and the coordinates are UTM.

the label $C_{n}=1$ ". Also it should be mentioned that there is no rule regarding suitability for a definite value of probability to specify that, by having this value of probability or greater, we will reach the assurance for relating an object to one specific class. But subjectively the probability equal to 0.7 $(70 \%)$ or more is good for relating an object to a specific class.

As seen in Table 9, the results of executing the codes on profile CD are not compatible with any of the 6 possible shapes of gravity sources (see Table 4). So the profile CD is not interpretable by SVC. Considering the location of CD profile in Fig. 7, we conclude that it is not a principal profile and it is not suitable for interpreting by SVC through our approach (see Sec- 


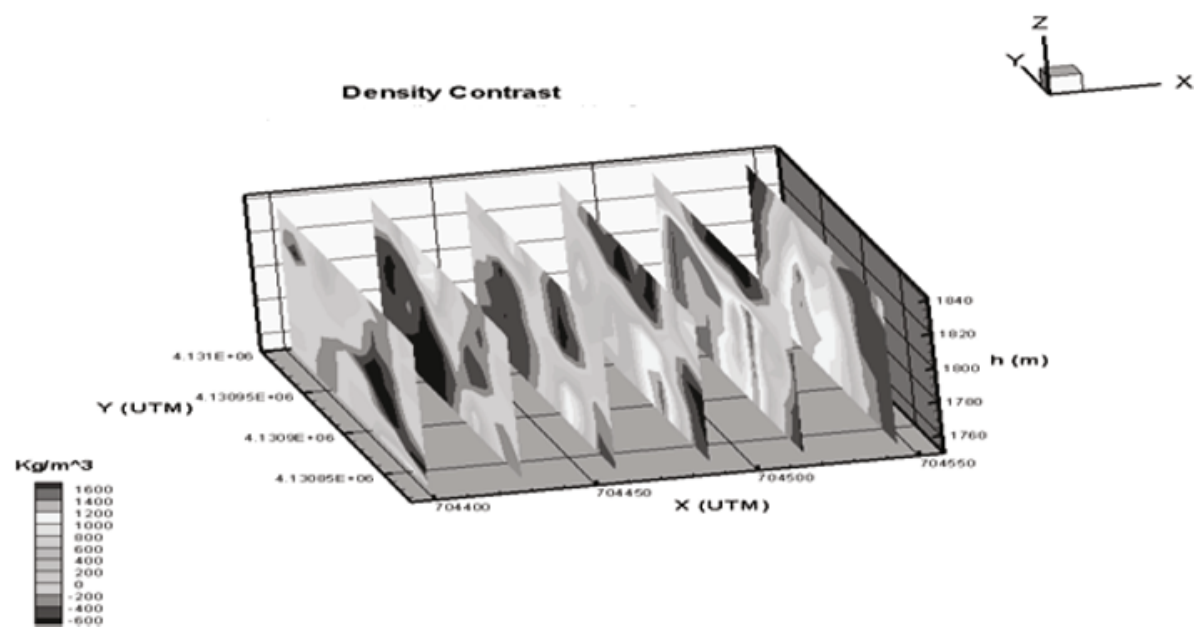

Fig. 8. The applied 3D inversion (based on the method of Camacho et al. 2002) on window 2 of Fig. 7. In the above figure, $h$ is the height above sea level.

tion 2.2). Here a question may arise why the probability of being related to any specific class for $\mathrm{CD}$ profile is high (see Table 9). The answer is that the classes of CD profile are estimated only according to the values of its features and these values are only meaningful if they are extracted from a principal profile.

The results of executing the codes on 3 other profiles ( $\mathrm{AB}, \mathrm{EF}$, and $\mathrm{GH})$ show that these profiles are probably related to rectangular prism shapes (see

Table 9

The results of executing the trained SVC codes related to the 4 classification groups (see point 5 of Section 3 ) on the real gravity profiles

\begin{tabular}{|l|l|l|l|}
\hline \multicolumn{4}{|c|}{ The results of executing the codes on profile } \\
\hline \multicolumn{1}{|c|}{$\mathrm{AB}$} & \multicolumn{1}{|c|}{$\mathrm{CD}$} & \multicolumn{1}{c|}{ EF } & \multicolumn{1}{c|}{ GH } \\
\hline $\begin{array}{l}\text { Limited shape } \\
\text { with probability 0.77 }\end{array}$ & $\begin{array}{l}\text { Elongated shape } \\
\text { with probability 0.99 }\end{array}$ & $\begin{array}{l}\text { Limited shape } \\
\text { with probability 0.77 }\end{array}$ & $\begin{array}{l}\text { Limited shape } \\
\text { with probability 0.77 }\end{array}$ \\
\hline $\begin{array}{l}\text { Up flat shape } \\
\text { with probability 0.85 }\end{array}$ & $\begin{array}{l}\text { Up flat shape } \\
\text { with probability 0.99 }\end{array}$ & $\begin{array}{l}\text { Up flat shape } \\
\text { with probability 0.85 }\end{array}$ & $\begin{array}{l}\text { Up flat shape } \\
\text { with probability 0.85 }\end{array}$ \\
\hline $\begin{array}{l}\text { Down flat shape } \\
\text { with probability 0.56 }\end{array}$ & $\begin{array}{l}\text { Down curved shape } \\
\text { with probability 0.84 }\end{array}$ & $\begin{array}{l}\text { Down flat shape } \\
\text { with probability 0.57 }\end{array}$ & $\begin{array}{l}\text { Down flat shape } \\
\text { with probability 0.57 }\end{array}$ \\
\hline $\begin{array}{l}\text { Non Circle section } \\
\text { shape with } \\
\text { probability } 0.58\end{array}$ & $\begin{array}{l}\text { Circle section } \\
\text { shape with } \\
\text { probability } 0.99\end{array}$ & $\begin{array}{l}\text { Non Circle section } \\
\text { shape with } \\
\text { probability } 0.58\end{array}$ & $\begin{array}{l}\text { Non Circle section } \\
\text { shape with } \\
\text { probability } 0.58\end{array}$ \\
\hline
\end{tabular}


Table 4). Regarding AB profile, we state that a vertical cylinder is very similar to vertical long rectangular prism and also the shape of the well inside the ground may in some places more resemble a vertical long rectangular prism than a vertical cylinder. Secondly, the results in Table 9 show that the shape of the source of $A B$ profile has a non-circle section shape with probability 0.58 , thus $\mathrm{AB}$ profile has a circle-section shape with probability equal to $1-0.58=0.42$ and the probability of having a vertical cylinder source shape is about equal to having a rectangular prism source shape. The conditions of profile $\mathrm{EF}$ are almost the same as profile $\mathrm{AB}$. To eliminate the ambiguity we tested these two profiles with 2 codes related to Table 8 . According to our tests on 24 codes of Table 8, two codes of the related 24 codes are the best ones for estimating the rectangular prism and the vertical cylinder. By using these 2 codes, the results are as follows:

$\checkmark$ By executing the one which is the most suitable for estimating the rectangular prism on profiles $\mathrm{AB}$ and $\mathrm{EF}$, the result was that with probability 0.18 the shapes of gravity sources of both profiles, $\mathrm{AB}$ and $\mathrm{EF}$, are rectangular prisms.

a By executing the one which is the most suitable for estimating the vertical cylinder on profiles $\mathrm{AB}$ and $\mathrm{EF}$, the result was that with probability 0.75 the shapes of gravity sources of profiles $\mathrm{AB}$ and $\mathrm{EF}$ are vertical cylinders.

- The results of executing the codes on profile GH show that this profile is related to rectangular prism gravity source shape with probability 0.7 .

The elongated shape of the gravity anomaly of GH profile (see Fig. 7) confirms that the shape of the gravity source cannot be a vertical cylinder. Finally, the estimated shapes of the gravity sources of the above-mentioned real gravity profiles by our different trained SVC codes are given in Table 10 .

Table 10

Shapes of gravity sources of the real gravity profiles and the results of their testing by our different trained SVC codes

\begin{tabular}{|c|l|l|}
\hline $\begin{array}{c}\text { Name } \\
\text { of the gravity profile }\end{array}$ & \multicolumn{1}{|c|}{$\begin{array}{c}\text { Shape } \\
\text { of gravity source }\end{array}$} & $\begin{array}{l}\text { Estimated shape of the source } \\
\text { by the trained SVC codes }\end{array}$ \\
\hline AB & Similar to vertical cylinder & Vertical cylinder \\
\hline $\mathrm{CD}$ & Possibly rectangular prism & $\begin{array}{l}\text { Not interpretable because of } \\
\text { non being principal profile }\end{array}$ \\
\hline $\mathrm{EF}$ & $\begin{array}{l}\text { Possibly vertical cylinder } \\
\text { or rectangular prism }\end{array}$ & Vertical cylinder \\
\hline $\mathrm{GH}$ & Possibly rectangular prism & Rectangular prism \\
\hline
\end{tabular}


We see that the estimation of the shapes of the real gravity sources with $\mathrm{SVC}$ is compatible with the reality.

\section{CONCLUSION}

The SVC was used for estimation of the gravity sources shapes. Also, the results were improved by optimizing SVC by selecting proper features.

Also, by increasing the dimensions of features space from 2 to 3 and 4 , we showed that among 375 subsets of features, where 210 subsets consisted of 4 features, only one subset, with 3 features, is the best. This means that increasing the dimensions of features space is sometimes not proper.

In this research, another important step was taken is developing several softwares that can test the results of each other. As you have seen in Table 6 (using the related trained codes) the highest portion of the corrected classified shapes in the testing set is related to the spherical shapes and the lowest portion is related to the rectangular prism shapes. Also, as you have seen in Table 7 (using the related 4 couples of codes) the highest portion of the corrected classified shapes in the testing set is related to both the horizontal cylinder and spherical shapes and the lowest portion is related to the syncline shapes. The related codes of Table 6 can be used independent of the related codes of Table 7 and vice versa. However, if we use all the above-mentioned codes together and if all the results confirm each other, the ill-posing of the gravity inversion will be treated relatively and results that are compatible among all the previously mentioned codes can be noticed approximately as the unique response. Therefore, we suggest that this approach can be applied for geoscientific investigations as a new approach for the estimation of the approximate shape of the gravity sources in exploration projects. We also suggest that this approach can be used in other branches of geophysics.

Acknowledgements. We thank all the faculty members who assisted us in preparing this paper. Our special thanks to Dr. Hashemi, for introducing us to the Pattern Recognition Laboratory, Delft University of Technology (whose group produced and designed PRTools which we used in this research). Also by studying his papers (Hashemi 2010, Hashemi et al. 2008), we decide to perform this research. We thank Prof. Duin (Pattern Recognition Laboratory, Delft University of Technology), for his helpful guidance at the start of this research. We thank the geophysics group of the Faculty of Basic Sciences of Science and Research Branch, Islamic Azad University, for guiding us so well during this research. Also we thank the Research Council of the University of Tehran. 


\section{References}

Ardestani, V.E. (2008), Modelling the karst zones in a dam site through microgravity data, Explor. Geophys. 39, 4, 204-209, DOI: 10.1071/EG08022.

Ardestani, V.E. (2009), Residual gravity map of a part of Institute of Geophysics of University of Tehran, University of Tehran, Tehran, Iran.

Baan, M., van der, and Ch. Jutten (2000), Neural networks in geophysical applications, Geophysics 65, 4, 1032-1047, DOI: 10.1190/1.1444797.

Belikov, M.V. (1978), Approximating of the external potentials of bodies rotation, M.Sc. Thesis on Physical-Mathematical Science, Institute of Theoretical Astronomy of the USSR Academy of Science, Leningrad State University (translation from Russian).

Blakely, J.R. (1996), Potential Theory in Gravity and Magnetic Applications, Cambridge University Press, Cambridge, $441 \mathrm{pp}$.

Camacho, A.G., F.G. Montesinos, and R. Vieira (2002), A 3-D gravity inversion tool based on exploration of model possibilities, Comput. Geosci. 28, 2, 191-204, DOI: 10.1016/S0098-3004(01)00039-5.

Duin, R.P.W., P. Juszczak, P. Paclik, E. Pekalska, D. de Ridder, D.M.J. Tax, and S. Verzakov (2007), PRTools4. 1 - A Matlab toolbox for pattern recognition, Delft University of Technology, Delft, Netherlands, http:/www. prtools.org/.

Gret, A.A., and E.E. Klingele (1998), Application of Artificial Neural Networks for Gravity Interpretation in Two Dimensions, Institute of Geodesy and Photogrammetry, Swiss Federal Institute of Technology, Zürich, Switzerland.

Hashemi, H. (2010), Logical considerations in applying pattern recognition techniques on seismic data: Precise ruling, realistic solutions, CSEG Recorder 35, 4, 47-50.

Hashemi, H., D.M.J. Tax, R.P.W. Duin, A. Javaherian, and P. de Groot (2008), Gas chimney detection based on improving the performance of combined multilayer perceptron and support vector classifier, Nonlin. Process. Geophys. 15, 6, 863-871, DOI: 10.5194/npg-15-863-2008.

Heijden, F., van der, R.P.W. Duin, D. de Ridder, and D.M.J. Tax (2004), Classification, Parameter Estimation and State Estimation, An Engineering Approach using Mathlab, John Wiley \& Sons Ltd, Chichester.

Hekmatian, M.E., V.E. Ardestani, M.A. Riahi, A.M. Koucheh Bagh, and J. Amini (2013), Fault depth estimation using support vector classifier and features selection, Appl. Geophys. 10, 1, 88-96, DOI: 10.1007/s11770-013-0371-7.

Osman, O., A.M. Albora, and O.N. Ucan (2006), A new approach for residual gravity anomaly profile interpretations: Forced Neural Network (FNN), Ann. Geophys. 49, 6, 1201-1208. 
Plouff, D. (1976), Gravity and magnetic fields of polygonal prisms and application to magnetic terrain corrections, Geophysics 41, 4, 727-741, DOI: 10.1190/ 1.1440645 .

Talwani, M., J.L. Worzel, and M. Landisman (1959), Rapid gravity computations for two-dimensional bodies with application to the Mendocino submarine fracture zone, J. Geophys. Res. 64, 1, 49-59, DOI: 10.1029/ JZ064i001p 00049.

Telford, W.M., L.P. Geldart, R.E. Sheriff, and D.A. Keys (1976), Applied Geophysics, Cambridge University Press, Cambridge.

Received 25 March 2013

Received in revised form 24 June 2014

Accepted 1 July 2014 